\title{
System Design of Electricity Generation Using Waste Heat from LNG Automobile
}

\author{
Canzong Zhou ${ }^{1}$, Shuyi Chen ${ }^{2}$, Wei Cui ${ }^{1}$ and Zhengmao $\mathrm{Yao}^{3}$ \\ ${ }^{1}$ Building electricity and intelligence, University of South China, Hengyang City, Hunan Province,421000, China \\ ${ }^{2}$ Building environment and energy engineering, University of South China, Hengyang City, Hunan Province,421000, China \\ ${ }^{3}$ English, Shanghai University of Sport, Shanghai City,200000, China
}

\begin{abstract}
According to the research, thermoelectricity generation can recycle the heat contained in the cooling system of internal combustion engine. This paper is about taking advantage of the feature in the huge temperature difference at about $560{ }^{\circ} \mathrm{C}$ which is formed between high-temperature engine and LNG (Liquefied Natural Gas) in low temperature and the ability that LNG provides semiconductor with thermoelectric conversion material so as to produce the maximum output voltage in low temperature. We take advantage of lead telluride materials that adapt to the high temperature environment and bismuth telluride materials that adapt to the low temperature environment, both of which forms a circuit and are designed as a thermoelectric power generation device. Also, we confirm the possibility of applying the device to cars.
\end{abstract}

\section{Introduction}

LNG is stored at a low temperature at about $-162^{\circ} \mathrm{C}$, and it needs to be heated from $-162^{\circ} \mathrm{C}$ liquid gasification to about $5^{\circ} \mathrm{C}$ for the use of automobile engine [1]. After the engine burns gaseous natural gas, a large amount of waste heat will be generated. The casual emission of engine waste heat not only pollutes the environment, but also causes a huge waste of energy. If this quantity of energy can be recycled, it will greatly improve the utilization of resources.

Now in foreign countries, the research of thermoelectric technology has aimed at the direction of practical application, and thermoelectric conversion efficiency has reached $10 \%$ in thermoelectric materials produced by some manufacturers, and some even as high as $15 \%$. The energy of the car in the waste heat recovery takes up about $7 \%$ of energy released from fuel burning, if this quantity of energy can be recycled to use in cars, the economical efficiency of fuel will increase by more than 20\% [2].Literature [3]-[4] indicates that the TEG (Thermoelectric Gemerator) system jointly developed by BMW and general motors has been tested for 50,000 kilometers on the Suburban hybrid vehicle, which shows that the system can save up to $10 \%$ of fuel consumption.

The earliest use of Thermoelectricity generation in auto engine was reported in 1997. Dong Guitian [5] of Jilin university of technology conducted an experiment about Thermoelectricity generation on Jiefang CA141 car engine. he designed a thermoelectric part using semiconductor lead telluride ( $\mathrm{PbTe}$ ) as material, in which the temperature difference can produce thermoelectromotive force $0.132 \mathrm{~V}$ at the hot end temperature at $500{ }^{\circ} \mathrm{C}$ and cold end temperature at $100{ }^{\circ} \mathrm{C}$. The success of the experiment shows that the waste heat thermoelectricity generator of automobile is enough to replace the traditional automobile generator, but the efficiency of this generation system is very low, only reaches about $3 \%$.

\section{Theoretical Calculation of Thermoelectric Power Generation}

\subsection{Theoretical Basis}

Thermoelectric power generation is to put p-type cavityrich material and n-type electron-rich material in high and low temperature sources respectively to form the PN junction, so that the internal free electrons and holes move in a directional manner under the influence of temperature, generating thermoelectric electromotive force and contact electromotive force, and forming a current in the loop [6]. This involves three different thermoelectric effects, the seebeck effect, the partie effect and the joule effect, which are closely related and mutually reinforcing.

\subsubsection{Seebeck Effect}

Seebeck effect, also known as thermoelectric first effect, refers to a closed loop composed of conductive materials of different properties, in which the electromotive force will be generated when the temperature of the combining position of the two materials is different [7].The 
thermoelectromotive force can be calculated by the following formula:

$$
V=\Delta T \times\left(\alpha_{P}-\alpha_{N}\right)
$$

In the formula:

$V$ _thermoelectric electromotive force generated by the thermoelectric circuit;

$\alpha_{P} \_$seebeck coefficient of p-type material;

$\alpha_{N} \_$seebeck coefficient of n-type material;

$\Delta T \_$temperature difference between hot end and cold end;

\subsubsection{Paltie Effect}

The paltie effect, also known as the thermoelectric second effect, refers to the phenomenon of heat absorption and heat release, in addition to joule heat, when the current passes through two different metal contact points. It is also known as the reverse reaction of seebeck effect. That is, when the current passes through the circuit composed of two different conductive materials, a temperature difference will be generated at the contact point [8]. If the direction of the current is changed, the phenomena of heat absorption and heat release will also change accordingly.

Among them, thermal power and current have the following relationship:

$$
Q=\Pi \cdot I
$$

In the formula:

Q__exothermic or endothermic power;

$\Pi$-_ parte coefficient is related to the properties of semiconductor thermoelectric power generation materials;

\subsubsection{Joule Effect}

The joule effect is one of the basic effects of electricity. The specific performance is: when there is electric current through the thermoelectric material, the material will produce a certain amount of heat. Its expression is:

$$
Q_{J}=I^{2} R
$$

In the Formula:

I- - the current passing through the material;

$\mathrm{R}$ - - resistance of the material;

The thermoelectric module is an important part of the thermoelectric device. The thermoelectric module adopted in this paper is composed of thermoelectric appliances and thermal insulation ceramic chips. The pterminal material of thermoelectric appliances is lead telluride $(\mathrm{PbTe})$, which has the best thermoelectric performance under high temperature environment. The n-terminal material is bismuth telluride ( $\mathrm{Bi} 2 \mathrm{Te} 3)$. A thermoelectric circuit is formed between the $\mathrm{P}$ end and the $\mathrm{N}$ end through a guide plate. The electrode materials used in the preparation of thermoelectric components should have good heat and electrical conductivity, and maintain good chemical stability with the semiconductor materials.

The data show that the physical and chemical properties of the semiconductor lead telluride and bismuth telluride are as follows:

Lead telluride was selected as a p-type semiconductor material, and its seebeck coefficient was $\alpha_{P}=380 \times 10^{-6} \mathrm{~V} / \mathrm{K} \quad, \quad$ The resistivity is $\rho_{P}=1.68 \times 10^{-3} \Omega \cdot \mathrm{cm}$, the Thermal conductivity is $K_{P}=1.499 \times 10^{-4} \mathrm{~W} / \mathrm{cm} \cdot K$, the length is $L_{P}=0.8 \mathrm{~cm}$, The cross-sectional area is $A_{P}=0.5024 \mathrm{~cm}^{2}$

Bismuth telluride was selected as an n-type semiconductor with seebeck coefficient as $\alpha_{N}=-270 \times 10^{-6} \mathrm{~V} / \mathrm{K}$, the resistivity is $\rho_{N}=1.0 \times 10^{-3} \Omega \cdot \mathrm{cm}$, the thermal conductivity is $K_{N}=2.4 \times 10^{-4} \mathrm{~W} / \mathrm{cm} \cdot K \quad, \quad$ the length is $L_{N}=0.8 \mathrm{~cm}$, the cross-sectional area is $A_{N}=0.5024 \mathrm{~cm}^{2}$

\subsection{Calculation of Electromotive Force of A Single Thermoelectric Unit}

A preliminary study on the power generation performance of semiconductor thermoelectric materials at low temperature found that the output electromotive force reached its maximum when the temperature at the cold end was between $-60^{\circ} \mathrm{C}$ and $-70^{\circ} \mathrm{C}$ [9].

The electromotive force generated by a single thermoelectric device can be calculated from the seebeck effect potential difference formula:

$$
\Delta U=\alpha_{P N} \cdot\left(T_{h}-T_{c}\right)
$$

In the formula:

$\Delta U$ - The electromotive force generated by a single thermoelectric device;

$\alpha_{P N}$ _the relative seebeck coefficient of p-type semiconductor material and n-type semiconductor material;

$T_{\mathrm{h}}$ _ the temperature of the hot end; the engine shall be used as the hot end; the temperature shall be $500^{\circ} \mathrm{C}$;

$T_{C} \_$cold end temperature: the LNG inlet shall be used as the cold end; the temperature shall be $-60^{\circ} \mathrm{C}$;

Each parameter is substituted into formula (4) to obtain: the electromotive force generated by a single thermoelectric device is $0.364 \mathrm{~V}$. 


\section{3 calculation of internal resistance of individual thermoelectric components}

The internal resistance of a single thermoelectric device can be calculated by the following formula:

$$
\mathrm{r}=\frac{\rho_{P} L_{P}}{A_{P}}+\frac{\rho_{N} L_{N}}{A_{N}}
$$

Put the parameters into the formula (5) to obtain the result: a single temperature electric resistance is 0.00427 $\Omega$.

\section{Feasibility Analysis of the Application in Automobiles}

Take Santana 2016 natural gas vehicle with $1.6 \mathrm{~L}$ emission as an example, calculate the thermal power generation efficiency of this thermoelectric device:

In order to meet the technical characteristics of the generator power $1080 \mathrm{~W}$, rated voltage $12 \mathrm{~V}$ and rated current $90 \mathrm{~A}$ configured by this car, and in combination with the maximum power transmission theorem of the circuit, 204 thermoelectric components mentioned above should be connected into 3 lines, each line should be connected in series with 68 to meet the impedance matching conditions, and the load can obtain the maximum power.

The thermoelectric power generation efficiency can be calculated as follows:

$$
\eta=\frac{P}{Q_{\mathrm{h}}}
$$

In the formula: $\mathrm{P}-$ - output power of thermoelectric power generation module;

$$
P=I^{2} R
$$

$\mathrm{R}-$ - the load resistance in the circuit (namely generator internal resistance), with a value of $0.133 \Omega$;

$Q_{\mathrm{h}}$ - - heat energy obtained from the thermal end of the thermoelectric power generation system;

$$
Q_{\mathrm{h}}=Q_{K}+Q_{P}-Q_{\mathrm{h}_{\mathrm{h}}}
$$

$Q_{K}$ - - Heat is transferred from the hot end to the cold end

$$
Q_{\mathrm{K}}=N\left(\frac{K_{P} A_{P}}{L_{P}}+\frac{K_{N} A_{N}}{L_{N}}\right)\left(T_{\mathrm{h}}-T_{c}\right)
$$

$\mathrm{N}-$ - Total number of thermoelectric components, $\mathrm{N}=204$;

$Q_{P}$ _ — the thermal end of the thermoelectric power generation module absorbs the heat of parscale;

$$
Q_{P}=\frac{I}{N_{2}} \times\left(\alpha_{P}-\alpha_{N}\right) \times T_{\mathrm{h}} \times N
$$

$\mathrm{N} 2--$ number of parallel thermoelectric device rows, $\mathrm{N} 2=3$;

$Q_{J h}$ __ joule heat released to the hot end by the thermoelectric power generation module;

$$
Q_{J h}=\frac{1}{2} I^{2} R_{1}
$$

The internal resistance of thermoelectric power generation system, whose value is $0.096 \Omega$

Substitute formula (7) - (11) into formula (6) to obtain:

$$
\eta=\frac{I^{2} R}{Q_{\mathrm{K}}+Q_{P}-Q_{J h}}
$$

Substituting the data obtained from 4.1-4.2 into formula (6) - (12), the efficiency of thermoelectric power generation device can be: $23.24 \%$.

The calculation does not take into account the inevitable heat loss during the actual operation and power semiconductor materials is negligible compared to the resistance of the electrode resistance influence on thermoelectric power generation efficiency, therefore, half of the theoretic efficiency is taken into account for actual efficiency, which is $11.62 \%$ as the proof of measuring thermoelectric power generation device for auto generator on the basis of electric energy supplement The results are enough to show that from the perspective of energy conversion relationship, the use of waste heat thermoelectric power generation can completely supply the electric energy provided by the automobile engine.

\section{Structure Design}

The thermoelectric power generation device designed in this paper is mainly composed of the following components: hot-end gas box (engine waste heat channel), cold-end gas box (LNG channel), thermoelectric power generation module group and clamping device. Its structure diagram is shown in figure 1. The thermoelectric power generation module group is installed between the hot-end gas box and the cold-end gas box to generate electricity by using the huge temperature difference at about $560^{\circ} \mathrm{C}$ formed by the high-temperature waste heat of the engine and the low temperature of LNG. After the high temperature waste heat discharged from the engine passes through the hotend gas box inside the thermoelectric power plant, the hot-end gas box is heated by conduction, convection and other heat transfer methods to make it warm. Through the cooling channel, LNG flows through the gas box at the cold end to significantly reduce the temperature, thus forming a huge temperature difference at both ends of the thermoelectric power generation module set, generating the required electric potential and providing 
the power output. The specific size and shape of the physical model are shown in figure 2. The hot end gas box and the cold end gas box adopt the more economic aluminum material with higher thermal conductivity. The overall structure of the system is shown in figure 3.



Figure 1 Schematic diagram of working principle of thermoelectric power generator
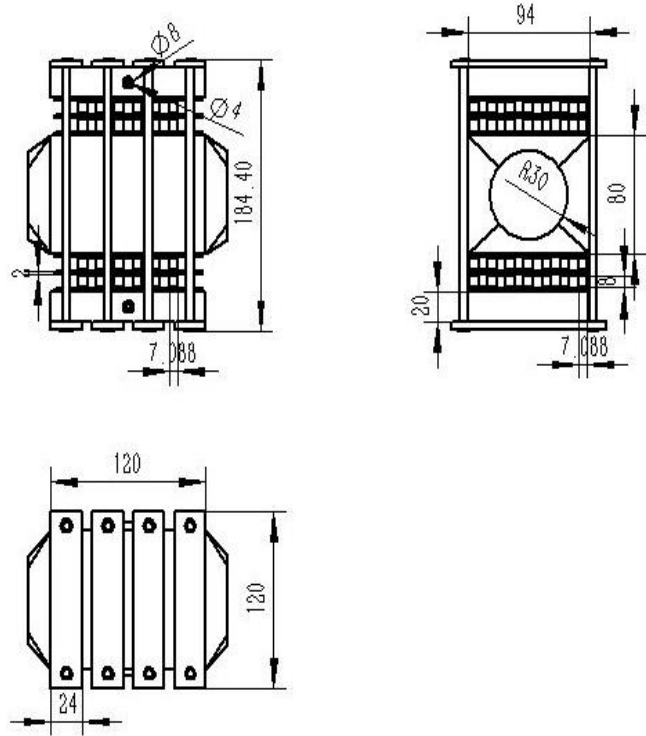

(1) Three view drawing



(2) The three dimensional figure

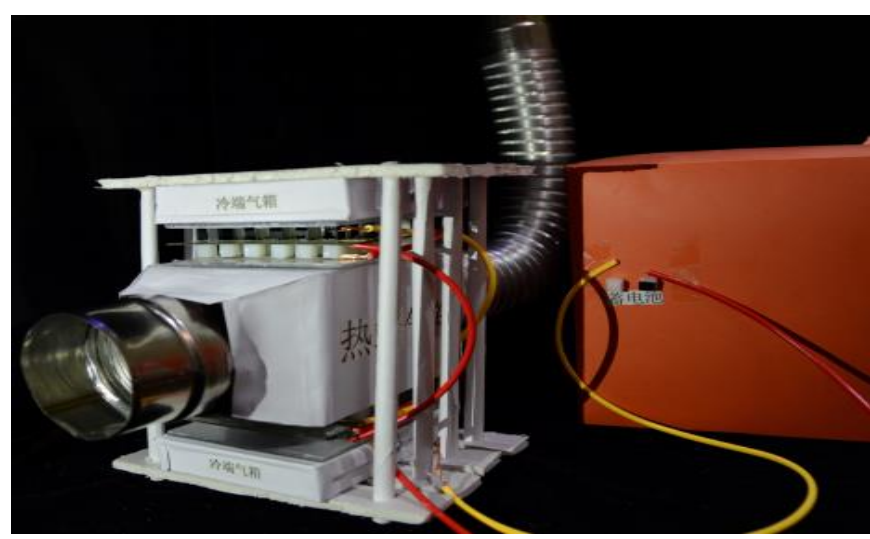

(3) model example

Figure 2 appearance of the work 


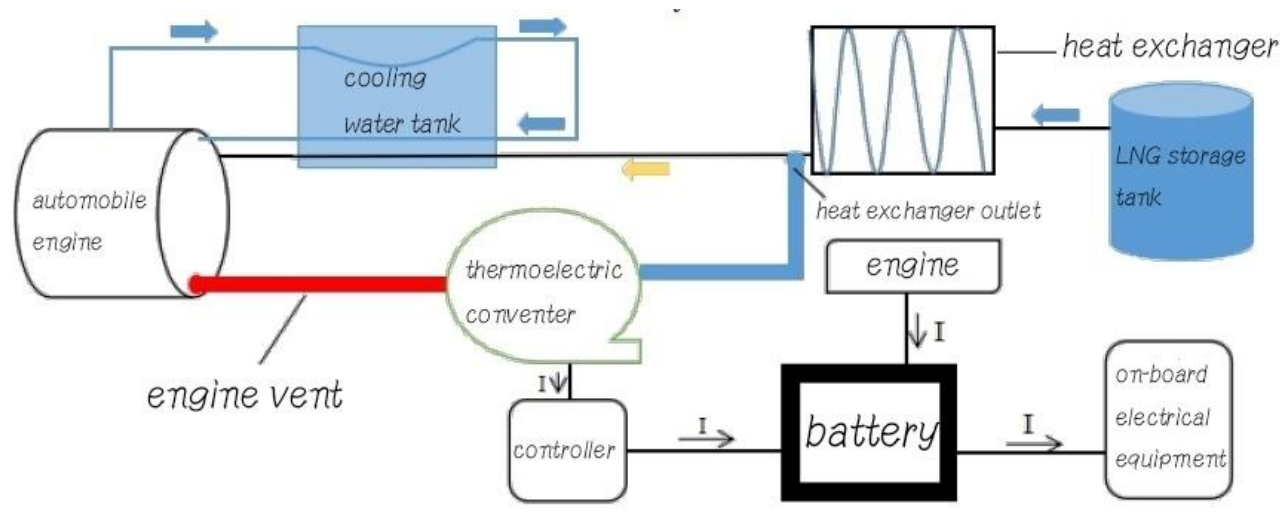

Figure 3 system overall structure diagram

\section{Application Prospect}

It can be obtained from formula (6) that, at the engine end of this santana car, the thermal power obtained by the thermoelectric power generator is $2797.25 \mathrm{w}$, in which the amount of converted electric energy reaches $2797.25 \times 11.62 \%=325.04(\mathrm{~W})$.

According to the literature, for every kilowatt $\cdot$ hour of electric power consumed during driving, an additional $1.145 \mathrm{Nm} 3$ of natural gas needs to be consumed. For every $1 \mathrm{Nm} 3$ of natural gas burned, $13.6 \mathrm{Nm} 3$ of carbon dioxide is released. From the above calculation, it can be seen that when the electric power that can be converted and stored in one hour of driving is $0.32 \mathrm{kw} \cdot$, saving natural gas $1.145 \times 0.33=0.378(\mathrm{Nm} 3)$, and carbon dioxide emission can be reduced by $0.378 \times 13.6=5.14$ (m3). It can be seen that its energy saving and emission reduction effect is very obvious.

The device has the following advantages:

(1) the device takes advantage of the low temperature characteristics of LNG to set the cold end temperature at the maximum output of potential temperature of the bismuth telluride material, so as to maintain its working performance within the optimal range.

(2) according to the working efficiency of semiconductor materials in different temperature segments, the thermoelectric power generation device is divided into high temperature segment and low temperature segment, and different thermoelectric materials capable of generating large electric potential are adopted in segments to improve their thermoelectric power generation efficiency.

(3) make use of the huge temperature difference between low-temperature LNG used for automobile engines and high-temperature waste heat of automobile engines to generate electricity, thus improving the efficiency of thermoelectricity generation.

(4) compared with traditional power generation methods, the thermoelectric technology adopted in this paper has the advantages of no noise, no vibration, no maintenance, no mechanical parts, and no need for liquid or gaseous media, so there is no environmental pollution problem.

The device is suitable for new energy vehicles fueled by LNG and can be used for:
(5) the car's own power reserve.

(6) normal power supply of automotive lighting, signal, electronic control system and auxiliary appliances

(7) supplement the power supply of the on-board electric appliances of the large motor home.

(8) power supply for car charger.

\section{Conclusion}

Through theoretical calculation and feasibility analysis, a new design scheme of thermoelectric power generation device is proposed in this paper. When the temperature of hot end is at $500^{\circ} \mathrm{C}$ and the cold end is at $-60^{\circ} \mathrm{C}$, the theoretical value of potential difference generated by a single thermoelectric device is $0.364 \mathrm{~V}$. The device adopts 204 thermoelectric components, which are connected into 3 lines with 68 units per line to meet the requirements of the 2016 santana of $1.6 \mathrm{~L}$ discharge. The vehicle requires a rated voltage of $12 \mathrm{~V}$ and rated current of $90 \mathrm{~A}$, and the efficiency will reach $11.62 \%$. The research and analysis in this paper show that the thermoelectric power generation device has certain feasibility in automobile and can improve the utilization of resources.

\section{References}

1. Wang Q. (2003) Research on air conditioning system of green automobile fuel - liquefied natural gas cooling recovery vehicle [D]. Xi 'an: xi 'an Jiaotong university.

2. Zhao F.D., Wang X.M, Liang R. etc. (2013) Status quo and key technology analysis of waste heat recovery from automotive thermal power [J]. Small internal combustion engine and motorcycle, 42 (2):94-96.

3. Yang J.H. (2009) Develop Thermoelectric Technology for Automotive Waste Heat Recovery[C]. DEER Conf., Dearborn.

4. Soonseo P., Jungho Y. (2009) Low and high Temprature Dual Thermoelectric Generation Waste Heat Recovery System for light-duty Vehicles[C].DEERConf., Dearborn. 
5. Dong G.T. (1997) Thermoelectric power generation from exhaust waste heat of automobile engine [J]. Beijing energy conservation, (4):7-9.

6. Zhan H. (2017) Research on automotive waste heat thermoelectric power generation system based on engine cooling system $[\mathrm{J}]$. Journal of Changchun university of engineering, 18 (3):58-62.

7. Zhang X. (2014) Theoretical research on thermoelectric power generation system based on automobile exhaust heat recovery [D]. Tianjin: tianjin university.

8. Bai J.H. (2015) Research on thermoelectric power generation system of cold energy and exhaust heat of liquefied natural gas (LNG) vehicles [D]. Taiyuan university of technology.

9. Jia L., Chen Z.S.,Sun W.( 2004)Journal of solar energy, 25 (4):443-446. 